\title{
Modeling Brain Growth and Development
}

\author{
Neda Sadeghi ${ }^{a}$, John H. Gilmore ${ }^{b}$, Guido Gerig ${ }^{a}$ \\ ${ }^{a}$ Scientific Computing and Imaging Institute, University of Utah, Salt Lake City, Utah 84112 \\ ${ }^{b}$ Department of Psychiatry, University of North Carolina, Chapel Hill, NC 27599
}

Keywords: Longitudinal brain imaging, early brain development, DTI, Nonlinear Mixed Effect Modeling

Many mental illnesses are thought to have their origins at early stages of development, encouraging increased research effort related to early neurodevelopment. Magnetic resonance imaging (MRI) has provided us with an unprecedented view of the brain in vivo. More recently, diffusion tensor imaging (DTI/DT-MRI), a magnetic resonance imaging technique, has enabled the characterization of the microstrucutral organization of tissue in vivo. As the brain develops, the water content in brain tissue decreases while protein and fat content increase due to processes such as myelination and axonal organization. Changes of signal intensity in structural magnetic resonance imaging (MRI) and diffusion parameters of diffusion tensor imaging (DTI) reflect these underlying biological changes.

Longitudinal neuroimaging studies provide a unique opportunity for understanding early brain maturation by taking repeated scans of individuals over the first two years. Despite the availability of anatomical images of the brain with unprecedented details, there has been little progress in accurate modeling of brain development or in creating predictive models of structures that could help identify early signs of illness. We have developed methodologies for the nonlinear parametric modeling of longitudinal structural MRI and DTI changes over the neurodevelopmental period to address this gap. This research provides a normative model of early brain growth trajectory as is represented in structural MRI and DTI data, which will be crucial to improved understanding of timing and potential mechanisms of atypical development. Growth trajectories are described via intuitive parameters related to delay, rate of growth and expected asymptotic values, all descriptive measures that can answer clinical questions related to quantitative analysis of growth patterns.

We demonstrate the potential of the framework with application to a study of early brain development of healthy controls (singletons and twins). Our framework is designed not only to provide qualitative comparisons, but also to give researchers and clinicians quantitative parameters and a statistical testing scheme. Moreover, the method includes modeling of growth trajectories of individuals, resulting in personalized brain maturation profiles.

The statistical framework also allows for prediction of subject-specific growth trajectories and confidence intervals, a new scheme which will be crucial for efforts to improve diagnosis for individuals and personalized treatment.

\section{Brain Growth as observed in anatomical MRI}

Magnetic resonance imaging (MRI) is appropriate for longitudinal pediatric studies since it does not use ionizing radiation and enables safe noninvasive scanning of young children. The brain undergoes significant changes during the first 2 years of life, with continued growth into adulthood. Previous cross-sectional neuroimaging studies have indicated an overall brain size increase during this period, reaching $80-90 \%$ of adult volume by age 2 Pfefferbaum et al. (1994). More recently, Knickmeyer et al. (2008) reported that total brain volume increases by $101 \%$ in the first year, followed by $15 \%$ in the second year. In addition to morphometric measures such as volume and shape, including cortical folding Xue et al. (2007); Knickmeyer et al. (2008); Huppi (2008); Murgasova et al. (2007), signal characteristics of brain tissue also change, reflecting the maturation of the underlying tissue. During the first 6 months after birth, the signal intensities of gray and white matter in T1-weighted (T1W) and T2-weighted (T2W) MR images are the reverse of those seen in adults. This is mainly due to the process of tissue myelination since white matter is mostly unmyelinated at birth. As white matter myelinates, signal intensity changes from hypointense to hyperintense relative to gray matter in $\mathrm{T} 1 \mathrm{~W}$ images. The reverse pattern is seen in $\mathrm{T} 2 \mathrm{~W}$ (from hyperintense to hypointense) as shown in Figure 1.

Myelination follows a spatiotemporal sequence as described by histological studies Yakovlev and Lecours (1967) and qualitatively by neuroradiologists Rutherford (2002). However, quantitative assessment of the maturation pattern of white matter is still lacking.

Development of cognitive functions is associated with white matter maturation, and hence abnormalities in observed diffusivity of white matter can be associated with cognitive deficits diagnosed in later life. 


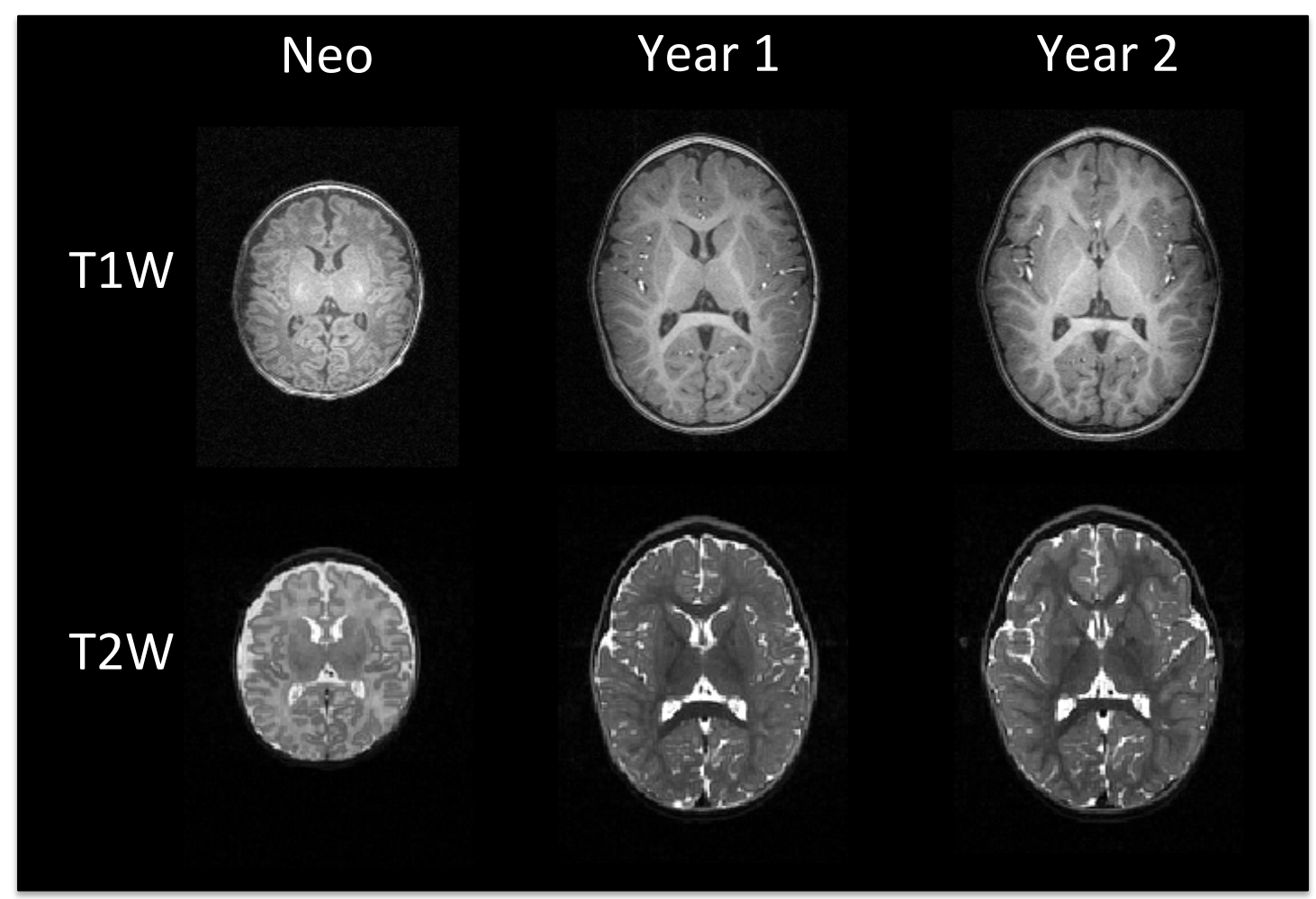

Figure 1: T1W and T2W images of an individual scanned at about 2 weeks, 1 year and 2 years. The pattern of brain gray and white matter contrast contrast at birth is the reverse of what is seen at 2 years.

\section{Early White Matter Maturation}

Diffusion tensor imaging (DTI) provides additional information about the microstructure of the brain. This method measures the average displacement of water molecules within tissue during a fixed time period. There is more diffusion where molecules can travel freely, and less diffusion where movement is impeded by obstacles such as cell membranes, myelin and macromolecules. Because the diffusion of water molecules is shaped by the underlying tissue structure, it is possible to gain an understanding of the underlying tissue structure by measuring diffusion. Fiber bundle organization can be depicted in DTI since water diffuses preferentally parallel to the fiber direction und to a lesseer extent in cross-sections. This anisotropic diffusion provides detailed information about brain axonal organization. As the white matter matures, diffusion of water molecules becomes more restricted as brain tissue undergoes structuring and myelination. Monitoring changes of diffusion parameters therefore provides information about the maturation pattern of white matter.

In diffusion tensor imaging, 3D motion of water molecules is modeled via a second order tensor at each voxel Basser et al. (1994). A tensor is represented as a diffusion matrix and can be visualized as an ellipsoid where the length of each primary axis represents average diffusion in each spatial direction Mori and Zhang (2006). The tensor information can be summarized by simpler in- variant quantitative measures (independent of the orientation of the reference frame) related to size or shape of the tensor. For example, one of the most common measurements is fractional anisotropy (FA), an index from 0 (isotropic) to 1 (anisotropic) indicating the shape of the tensor ranging from a sphere to a thin stick Pierpaoli and Basser (1996). Another measurement is mean diffusivity which can be explained by the average length of axes of the ellipsoid indicating the size of the tensor. This measure has proved useful for assessing the diffusion drop in brain ischemia van Gelderen et al. (1994), for example. More recently, axial diffusivity (AD) and radial diffusivity (RD) have been proposed to help to better understand the changes of the diffusion tensor Sadeghi et al. (2010); Alexander et al. (2007). AD is the length of the longest axes of ellipsoid indicating the fiber orientation and RD is the average of the two shorter axes. Analysis of DTI data of pediatric subjects has illustrated changes of these indices due to development Gilmore et al. (2007); Dubois et al. (2006). Cascio et al. found overall increases in fractional anisotropy during development and reduced overall diffusion due to development Cascio et al. (2007).

FA values can also be color-coded by using the direction of the main axis of the local tensor ellipsoid. Red is used to indicate the left-right direction, blue is used for superiorinferior, and green for anterior-posterior directions Pajevic et al. (1999). Figure2 shows color-coded FA images of one 


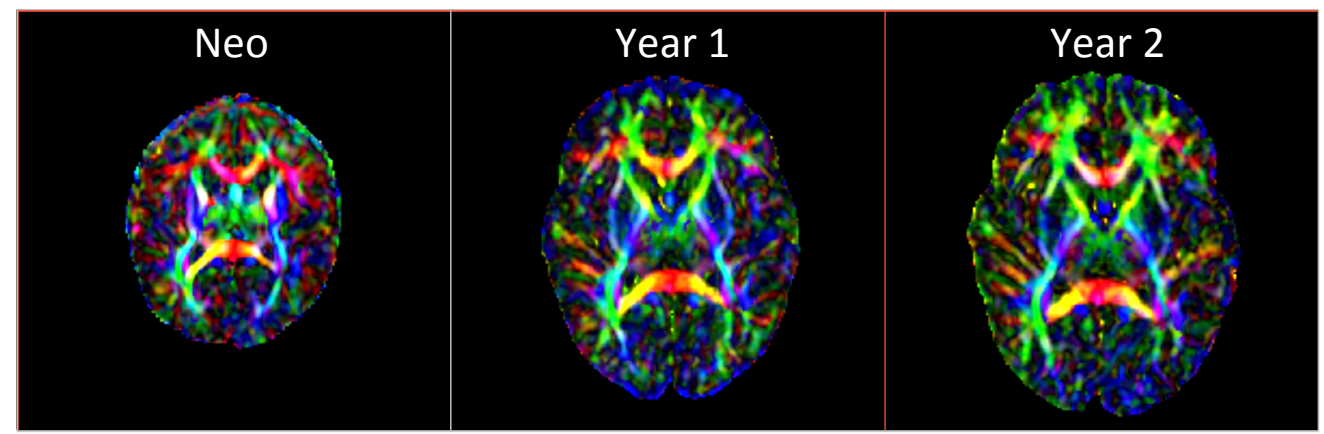

Figure 2: Color-coded fractional anisotropy (FA) images of an individual subject. Left to right: scans at 2 weeks, 1 year and 2 years.

subject at 2 weeks, 1 year and 2 years. The brightness is weighted by the fractional anisotropy Pajevic et al. (1999).

\section{Objectives of Longitudinal Neuroimaging Studies}

The defining feature of longitudinal studies is that subjects are measured repeatedly over the course of the study. This is in contrast to cross-sectional studies in which an individual is measured at only one single time-point. Longitudinal studies enable assessment of within-individual changes in the response variable, and thereby have the capacity to separate between cohort and age effects. The main aim of a longitudinal study is the characterization of within-individual changes over time and to determine whether within-individual changes in the response are associated with specific covariates such as treatment plan, clinical group or biological factors.

The main characteristic of longitudinal data is the correlation among repeated measurements. As these measurements are obtained on the same individual, there is a correlation among the measurements, with measurements obtained closer in time being more correlated than the ones further apart. This correlation among repeated measurements violates the fundamental independence assumption of most statistical regression techniques Fitzmaurice G.M. (2011) and requires different analysis schemes.

Most longitudinal studies plan to obtain the same number of measurements for each individual at the same time points; however, this is difficult to achieve in clinical practice. With studies that span over a longer period of time, it is inevitable that some individuals will drop out of the studies and some might miss their appointments and are rescheduled for different time points. The statistical analysis method as described in the following can appropriately handle uneven spacing of time points and also cope with missing data which can also be caused by exclusion of image data due to subject motion.

\section{Longitudinal Pediatric Neuroimaging Studies}

Understanding early brain development has great scientific and clinical importance. The human brain undergoes rapid organization and structuring early in life, and also there is great heterogeneity among different individuals. Longitudinal modeling of longitudinal data yields a more accurate average trajectory over time without the confounding cohort effects Diggle et al. (2002); Fitzmaurice G.M. (2011). This is of great importance when the development itself is in question. Recently, longitudinal image data have become available for the critical period of development just after birth. However, normative models are still not available to describe the normal pattern of development as shown in structural and diffusion MRI.

By designing appropriate longitudinal statistical analysis, we can model the average trajectory via a parametric function which can summarize growth with a few parameters. This also enables comparison of a normative population model to other groups or to individuals. For example, we can model population changes of subjects who have been diagnosed with a specific disease and compare this growth curve to the normative model to gain a better understanding of the pathology and to the time when deviation occurs. We can also gain a better understanding of the spatio-temporal sequence of maturation of white matter in the developing brain.

Once average trajectories for different groups are obtained, we can make inferences about parameters of the regression. In this work, we consider studying longitudinal changes of diffusion parameters of DTI for a group of infant subjects $(\mathrm{N}=26)$ from 2 weeks to 2 years old to establish a normative pattern of development along with its variability. The parametric Gompertz function is used to characterize these changes over time as it uses intuitive parameters describing growth: asymptote, delay and speed. As a proof of concept, white matter regions that are known to mature at different rates are analyzed and compared. We also applied the methodology to estimate developmental trajectories for twins and singletons and compare these trajectories between the two groups. We hypothesized that there may be group differences between developmental trajectories of twins and singletons due to differences in preand postnatal environments. 

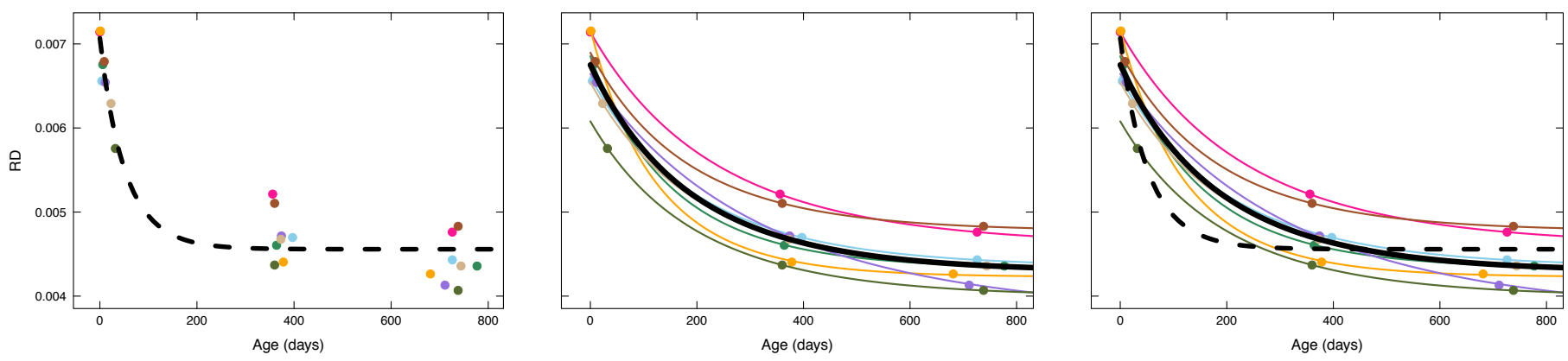

Figure 3: Population growth models, represented as dashed black curves, obtained using nonlinear least squares (NLS) on the left and with nonlinear mixed effect modeling (NLME) in the middle. Colored points represent data observations, and colored curves represent the individual growth trajectories. NLME (solid black) better models how individuals progress on average if compared to NLS (dashed black), with overlay of both shown on the right.

\section{Growth Models}

Brain growth functions clearly show the nonlinear nature of changes, starting with rapid changes which flatten off with increasing age. This characteristics can be observed in measurements of brain volumes, of head circumference, tissue contrast in T1 or T2 weighted anatomical MRI, but also in white matter diffusion obtained from DW-MRI. Linear modeling therefore cannot capture this structure and have to be replaced by nonlinear growth modeling. Nonlinear models of growth are generally based on a differential equation relating growth rate $d y / d t$ to response variable $y$ (i.e., size, diffusion parameter) Karkach (2006). This formulation has led to a variety of growth models, uch as exponential, monomolecular, logistic and Gompertz functions. We have demonstrated in Sadeghi et al. (2013) that the parametric Gompertz function provides best suits the purpose of modeling early brain development. Moreover, the parameters of the Gompertz function also provide intuitive parameterization of growth in terms of asymptotic value, delay and growth rate.

$$
y=\text { asymptote } \exp (- \text { delay } \exp (- \text { speed } t))
$$

\section{Nonlinear Mixed Effects Model}

Mixed effects models provide a powerful and flexible environment for analyzing longitudinal data, properly accounting for the intercorrelation among observations on each subject Diggle et al. (2002). In the mixed effects model, the observed data are assumed to be a combination of both fixed effects, $\beta$, parameters associated with the entire population (or at least within a subpopulation), and random effects, $b$, that are specific to an individual drawn at random from the population. A mixed effects model distinguishes between a within-subject source of variability and a between-subject source of variability. Correlation among repeated scans of an individual is accounted for by incorporating random effects in the model. Mixed effects model also results in a group trend (fixed effects) that better reflect how individuals progress on average compared to a least square fit as is shown in Figure 3.

\section{Regional Characterization}

\section{Normative Models for White Matter Diffusivities}

The nonlinear mixed effects are used to model the longitudinal changes of diffusion parameters within anatomical regions of interest. A white matter label map developed and disseminated by Mori et al. (2008) was used to define regions of interest in our infant image data set. We select 13 anatomical regions in the atlas space as shown in Figure 4. In this study, left and right regions of anatomical locations are combined, giving a total of eight regions. Future studies on lateralization of growth differences will analyze left and right regions separately. The labeling of regions in the atlas space allows automatic partitioning of each subjects' scans into the different anatomical regions as all the subjects have been mapped to the atlas space (for details on the image processing pipeline please see Sadeghi et al. (2012)). We then estimate growth trajectories for these regions using the NLME model of Lindstrom and Bates (1990). Figure 4 illustrates the average FA for each region. In all the regions, FA increases with age, however, each region has its own distinct temporal pattern. Most of the regions show a rapid growth in the first year with continued growth but at a slower rate in the second year, however, genu of corpus callosum shows a steady growth during the first two years. Genu is one of the regions that develops later and its maturation seems to continue into developmental stages later than the two years' observation period.

\section{Hypothesis Testing}

Parameter estimation of NLME is based on the maximum likelihood of marginal density of responses. The distribution of maximum likelihood of the fixed effects is approximated by a normal distribution. Knowing fixed effects and its sampling distribution, approximate confidence intervals of fixed effects can be calculated and hypothesis testing can be performed between regions of interest. 

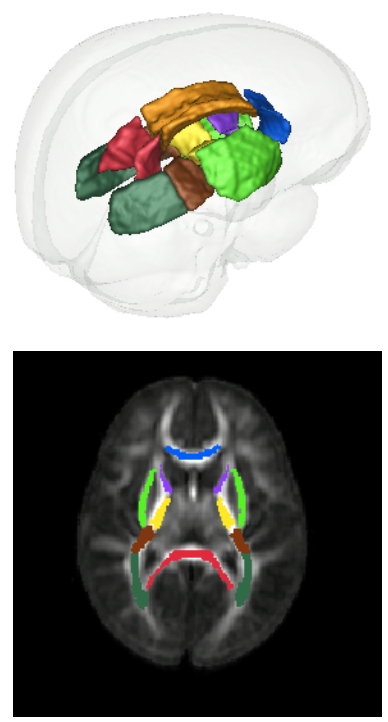

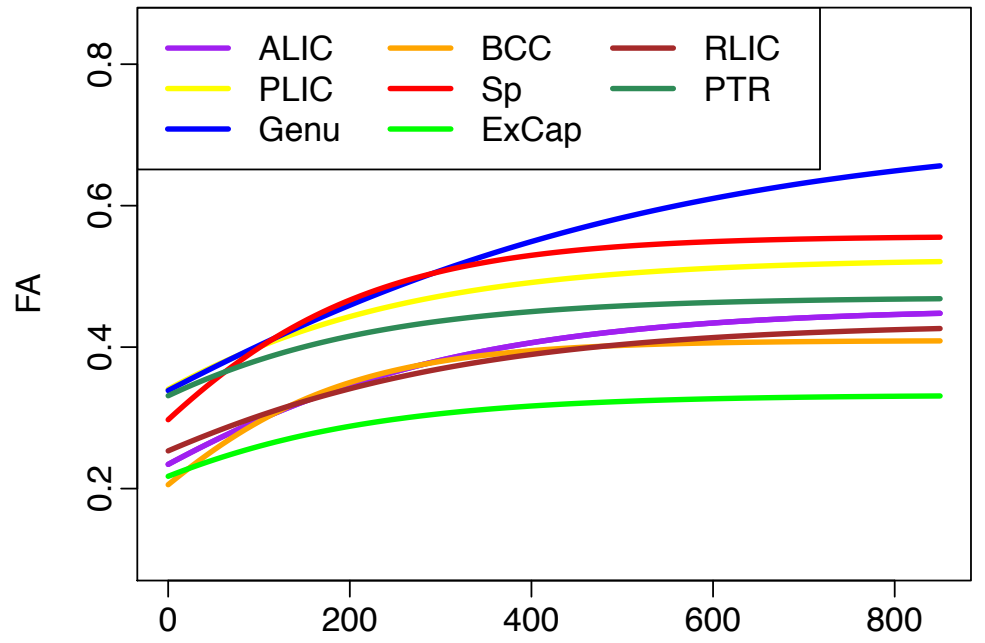

Age (Days)

Figure 4: Average growth trajectories of anterior limb of internal capsule (ALIC), posterior limb of internal capsule (PLIC), genu, body, and splenium of the corpus callosum (Genu, BCC, and Sp), external capsule (ExCap), retrolenticular part of the internal capsule (RLIC) and posterior thalamic radiation are shown. FA values increases for all these regions during early brain development, but different structures depict a distinct spatio-temporal pattern.

We conduct hypothesis testing between pairs of regions to determine modes of longitudinal changes in terms of the Gompertz growth parameters. With $\mathrm{N}$ number of regions, we perform $\frac{N(N-1)}{2}$ pairwise fitting of nonlinear mixed effect modeling. The significant parameters are determined through t-tests, corrected for multiple comparisons by Bonferroni correction. The parameters that are found to be significant between two pairs of regions can be interpreted as the distinguishing feature between the longitudinal trajectories of these regions. Figure 5 shows population and individual growth trajectories for FA and RD of genu (shown in blue) compared to splenium (shown in red). The anterior region of corpus callosum is genu with tracts ending in prefrontal cortex, whereas splenium is the posterior region with tracts ending in occipital lobe. Both of these regions are unmyelinated at birth and develop quickly during the first two years of life. Overall, genu shows higher $\mathrm{MD}, \mathrm{RD}$, and $\mathrm{AD}$ during the first two years while FA shows higher values mostly for the second year. This suggests genu is less mature at birth but develops rapidly reaching same level of $\mathrm{MD}$ and $\mathrm{RD}$ as splenium by the second year. Both genu and splenium have relatively higher $\mathrm{AD}$ compared to other regions of the brain indicating higher axonal organization for these regions.

\section{Inference and Prediction}

In addition to modeling the mean trajectory of diffusion properties over time and hypothesis testing among different regions, another important aspect of longitudinal analysis is the direct estimation of intra-individual changes over time. Even if not all observations for all time points are available for a subject, by pooling the data from other subjects in the study along with the available observations for the individual, prediction of an individual trajectory is possible. The estimation of personalized growth profiles is of significant clinical interest as individuals respond differently to treatment and show different growth trajectories. The figure below shows the approximate subject growth trajectory along with the subject-specific prediction interval for RD values of an individual based on the available scans at neonate and 1 year using NLME. The gray shaded region in the figure shows the prediction interval based on the population parameters and variability among individuals, whereas the blue region indicates the subject-specific prediction interval based on the available population parameters and the new individuals' available data. Also, in cases when only a single scan is available, the intensity or diffusion parameters of the subject can be compared to the normative model to indicate whether an individual is within the normative range of variability. Using such an estimation scheme, subject-specific growth trajectory and predictive intervals can be predicted based on only one scan. Such predictions might improve early detection and outcome since the subject-specific prediction interval not only accounts for the population estimated parameters but also considers the new individual's available data.

\section{Brain Maturation Differences Singletons versus Twins}

Twin studies have provided a valuable insight into the heritability of disease; however, it might be difficult to generalize these findings to a singleton population due to differences between twins and singletons in pre- and postnatal environments Knickmeyer et al. (2011); Hulshoff Pol 


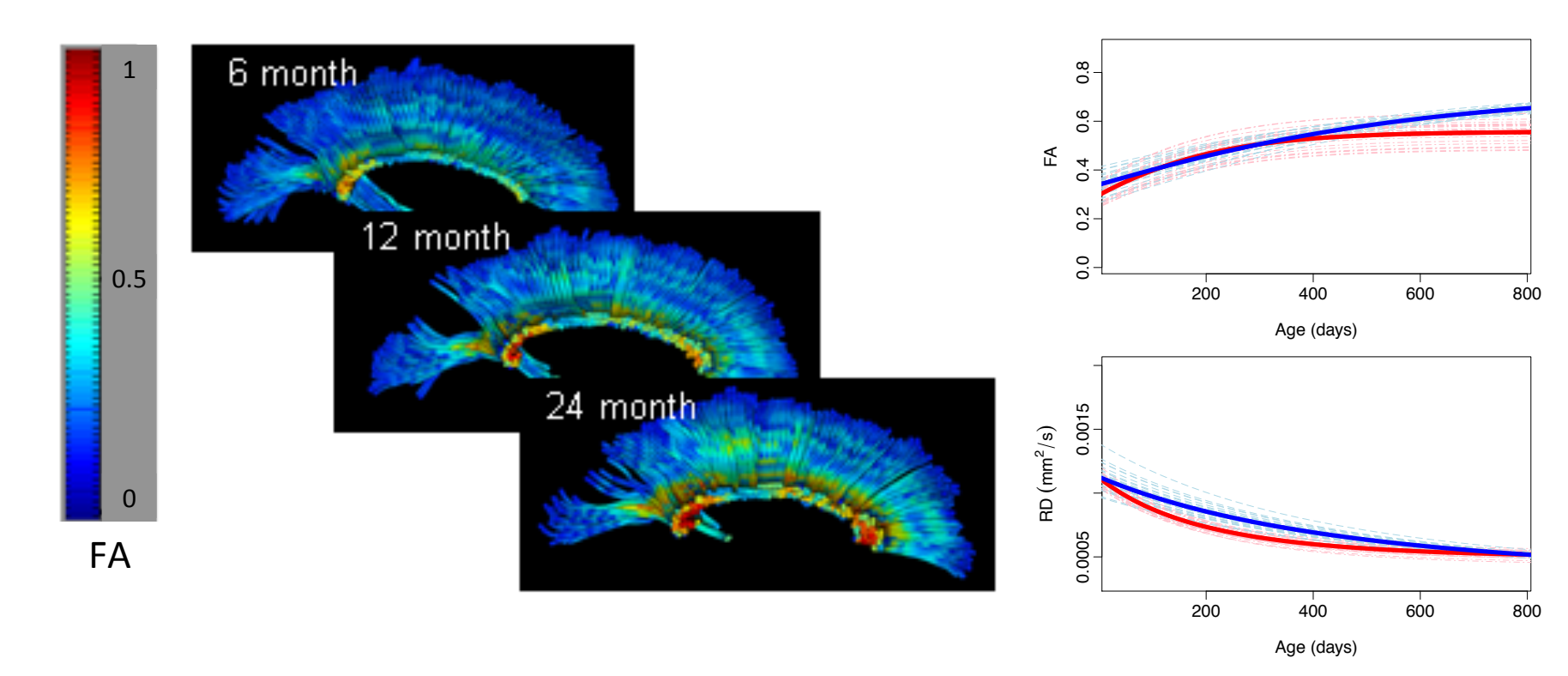

Figure 5: Left: Color FA of corpus callosum at 6, 12, and 24 months (left side of the image is the posterior region (splenium) whereas the right side is the anterior region (genu) of cc). Right: Population and individual growth trajectories for genu (blue) and splenium (red). Thick curves are the average growth trajectories, whereas dashed curves are individual trajectories. The following Gompertz parameters where significantly different $(p<0.05)$ between these two regions: FA: asymptote and speed, RD: speed.

et al. (2002). The intrauterine environment might be suboptimal as twins share the womb and compete for nutrition. Also, the family environment can be suboptimal due to limited resources and competition between the twins Hay and O'Brien (1983).

A recent study by Knickmeyer et al. found significant differences in gray matter development in MZ twins compared to DZ twins and singletons, but no differences were found in intracranial volume, total white matter volume and lateral ventricle volume Knickmeyer et al. (2011). In this study we examine the compatibility of white matter developmental trajectories between twins and singletons.

Comparison of mean trajectories among monozygotic (MZ), dizygotic (DZ) and singletons indicated that growth trajectories of monozygotic and dizygotic twins are very similar. No significant differences were found between the growth curves of MZ and DZ in terms of Gompertz parameters of asymptote, delay and speed for any of the diffusion measurements. To further investigate whether twins and singletons show any developmental differences, DZ and MZ individuals were combined as there were no differences in their growth trajectories. Gestational age was controlled in the analysis as twin subjects are generally born earlier than singletons, in our study by three weeks. When comparing the combined twin group to singletons, the following regions showed significant differences in the delay parameter of the axial diffusivity measures: right and left anterior limb of the internal capsule and right and left anterior corona radiata (Figure 8). There were no significant differences in asymptote and speed parameters between these two groups for any of the regions analyzed. There were also no significant differences between FA and RD measures between these two groups. These preliminary findings suggest that twins and singletons follow similar growth trajectories for most white matter regions. This study compared 21 anatomical regions, including projection fibers such as internal capsule and corona radiata, association fibers including superior longitudinal fasciculus and external capsule, and commissural fibers such as genu, body and splenium of corpus callosum. Fractional anisotropy and radial diffusivity did not differ between twins and singletons in all the regions that were analyzed after correction for multiple comparisons. However, twins and singletons did exhibit differences in axial diffusivity measures in the anterior limb of the internal capsule and the anterior region of the corona radiata. There were significant differences in the delay parameter of the Gompertz function for these regions, indicating that twins were delayed compared to singletons. However, twins appear to have caught up to singletons by 3 to 4 months postterm as though they experience a period of catch-up growth postbirth (Figure 8). There were no significant differences in the asymptote parameter of the Gompertz function, suggesting that the twin-singleton differences observed early on in these regions disappear by early childhood.

\section{Acknowledgments}

This research was supported by NIH grants: R01 MH070890 (JHG, GG), Conte Center MH064065 (JHG,GG), and National Alliance for Medical Image Computing (NA-MIC) U54 EB005149 (GG).

\section{Relevant Websites}

Utah Center for Neuroimage Analysis www.ucnia.org 

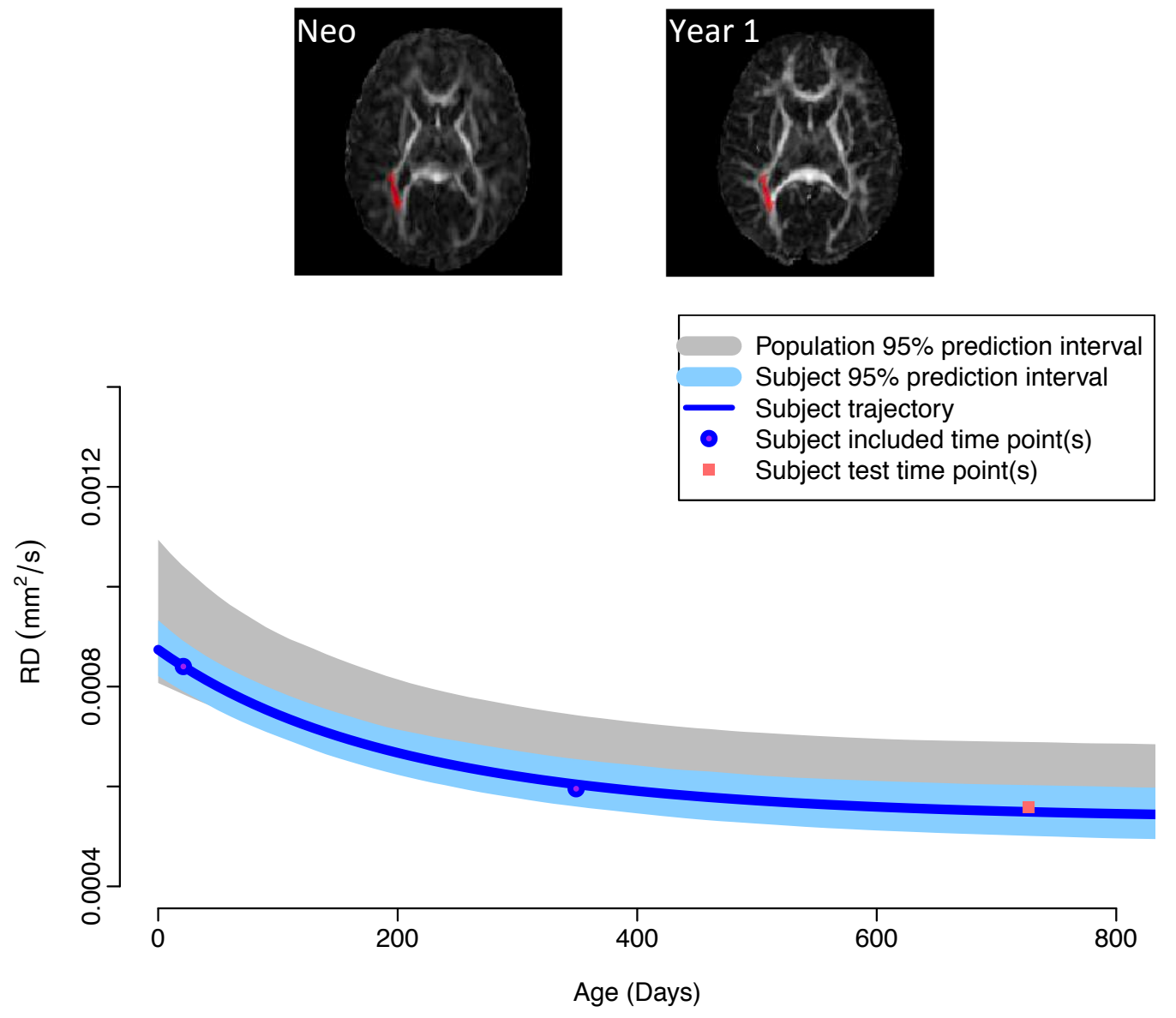

Figure 6: Top: Posterior thalamic radiation is shown as red label on the neonate and year 1 FA scans of one subject. Bottom: Subject $95 \%$ prediction interval compared to the overall prediction for RD of posterior thalamic radiation. Subject-specific interval calculated based on the subject's scans at neonate and 1 year in addition to population parameters. Subject's year 2 RD value (red square) falls within the predicted range (blue region).

Encyclopedia on early childhood development: Brain Development: http://www.child-encyclopedia.com/ en-ca/child-brain/according-to-experts.html

Carolina Institute for Developmental Disabilities: http: //www.cidd.unc.edu/piven/

Conte Center for Schizophrenia: https://www.cidd. unc.edu/research/default. aspx?id=45

Brain Development in Autism: http://www.
ibisnetwork.org/

\section{References}

Alexander, A. L., Lee, J. E., Lazar, M., Boudos, R., DuBray, M. B., Oakes, T. R., Miller, J. N., Lu, J., Jeong, E.-K., McMahon, W. M., et al., 2007. Diffusion tensor imaging of the corpus callosum in autism. Neuroimage 34 (1), 61-73.

Basser, P. J., Mattiello, J., LeBihan, D., 1994. Mr diffusion tensor spectroscopy and imaging. Biophysical journal 66 (1), 259-267.

Cascio, C. J., Gerig, G., Piven, J., 2007. Diffusion tensor imaging: application to the study of the developing brain. Journal of the American Academy of Child \& Adolescent Psychiatry 46 (2), 213223.

Diggle, P., Heagerty, P., Liang, K., Zeger, S., 2002. Analysis of Longitudinal Data, 2nd Edition. Oxford University Press, New York.
Dubois, J., Hertz-Pannier, L., Dehaene-Lambertz, G., Cointepas, Y., Le Bihan, D., May 2006. Assessment of the early organization and maturation of infants' cerebral white matter fiber bundles: a feasibility study using quantitative diffusion tensor imaging and tractography. NeuroImage 30, 1121-1132.

Fitzmaurice G.M., Laird N.M., W. J., 2011. Applied Longitudinal Analysis, 2nd Edition. John Wiley \& Sons, New Jersey.

Gilmore, J., Lin, W., Corouge, I., Vetsa, Y., Smith, J. K., Kang, C., Gu, H., Hamer, R., Lieberman, J., Gerig, G., 2007. Early postnatal development of corpus callosum and corticospinal white matter assessed with quantitative tractography. American Journal of Neuroradiology 28 (9), 1789-1795.

Hay, D. A., O'Brien, P. J., Apr 1983. The La Trobe Twin Study: a genetic approach to the structure and development of cognition in twin children. Child Dev 54 (2), 317-330.

Hulshoff Pol, H. E., Posthuma, D., Baare, W. F., De Geus, E. J., Schnack, H. G., van Haren, N. E., van Oel, C. J., Kahn, R. S., Boomsma, D. I., Feb 2002. Twin-singleton differences in brain structure using structural equation modelling. Brain 125 (Pt 2), 384-390.

Huppi, P., Oct 2008. Neuroimaging of brain development-discovering the origins of neuropsychiatric disorders? Pediatr Res 64,325 .

Karkach, A., 2006. Trajectories and models of individual growth. Demographic Research 15 (12), 347-400.

URL http://www.demographic-research.org/volumes/vol15/ $12 /$

Knickmeyer, R., Gouttard, S., Kang, C., Evans, D., Wilber, K., 

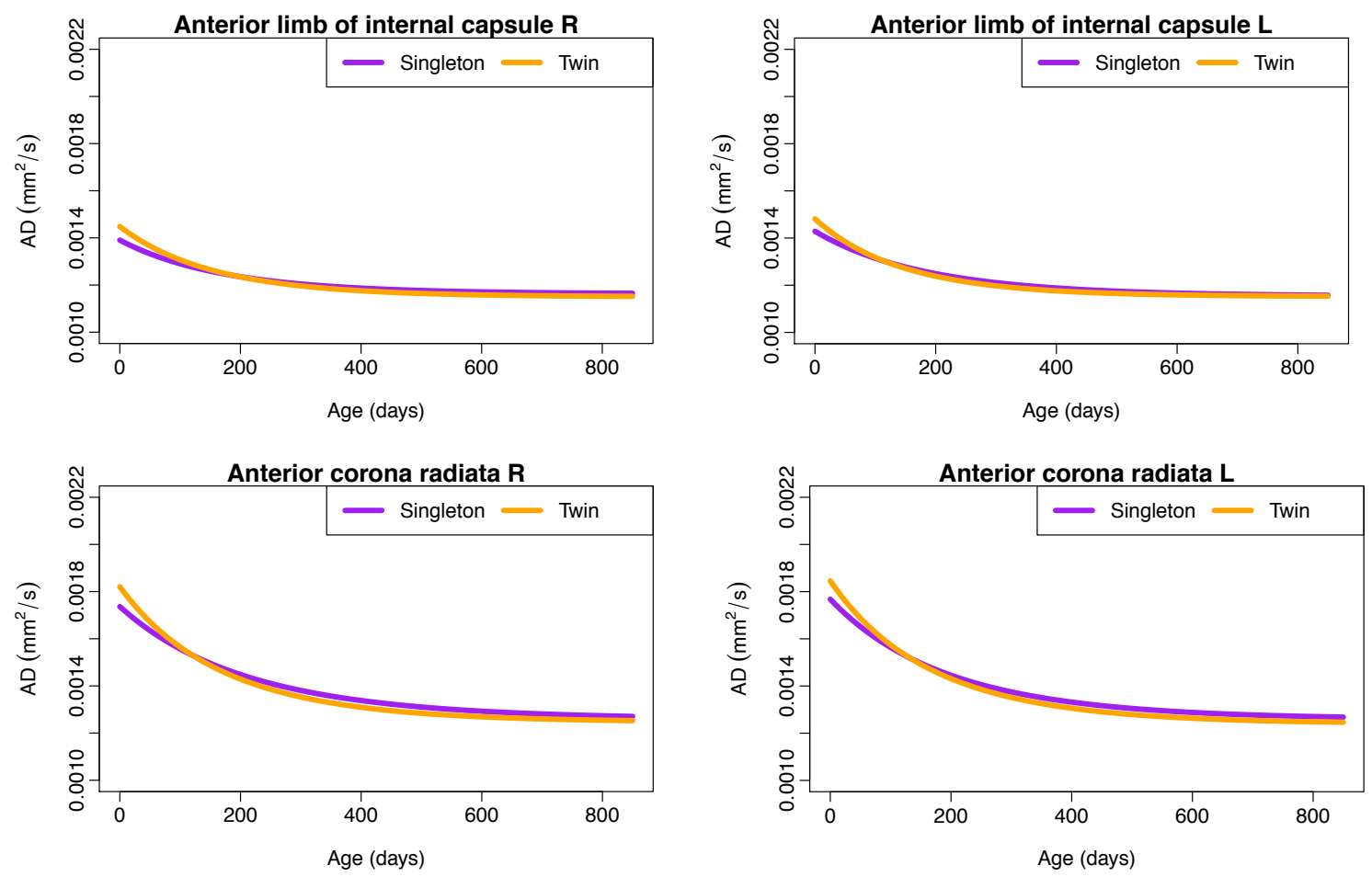

Figure 7: Comparison of $\mathrm{AD}$ growth trajectories of twins and singletons for the anterior limb of the internal capsule and the anterior corona radiata. The delay parameter $(p<0.05)$ was significantly different between twins and singletons in these two regions.
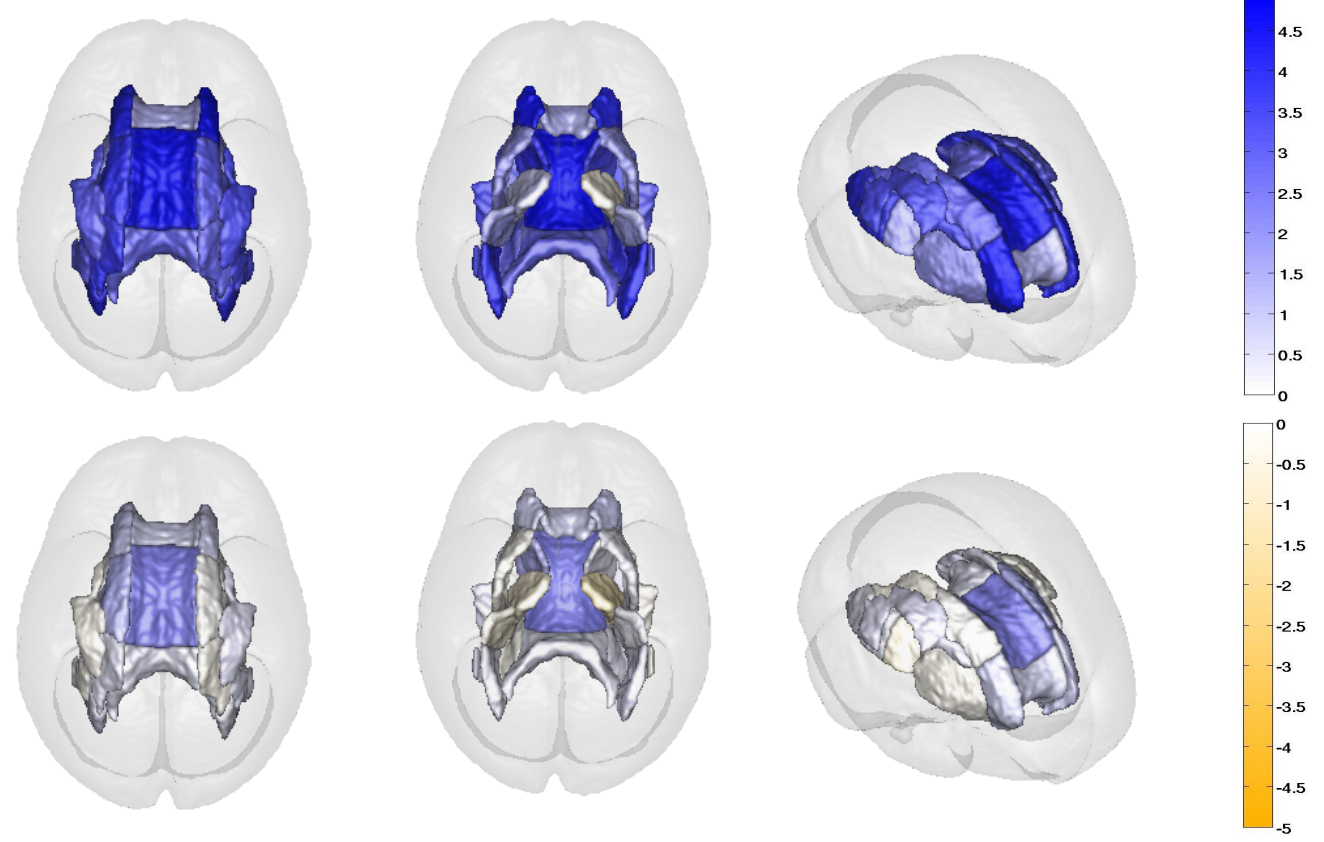

Figure 8: Differences in axial diffusion (AD) of Twins vs. Singletons ((Twin - Singleton)/Singleton)*100. Top: Differences in AD between twins and singletons at birth. Bottom: Differences at 3 months. The changes from dark blue at birth to light blue at 3 months indicate that differences between twins and singletons quickly become much smaller reach the same white matter maturation values. 
Smith, J., Hamer, R., Lin, W., Gerig, G., Gilmore, J., Nov 2008. A structural MRI study of human brain development from birth to 2 years. J Neurosci $28,12176-12182$.

Knickmeyer, R. C., Kang, C., Woolson, S., Smith, J. K., Hamer, R. M., Lin, W., Gerig, G., Styner, M., Gilmore, J. H., Jun 2011. Twin-singleton differences in neonatal brain structure. Twin Res Hum Genet 14 (3), 268-276.

Lindstrom, M., Bates, D., Sep 1990. Nonlinear mixed effects models for repeated measures data. Biometrics 46, 673-687.

Mori, S., Oishi, K., Jiang, H., Jiang, L., Li, X., Akhter, K., Hua, K., Faria, A., Mahmood, A., Woods, R., Toga, A., Pike, G., Neto, P., Evans, A., Zhang, J., Huang, H., Miller, M., van Zijl, P., Mazziotta, J., Apr 2008. Stereotaxic white matter atlas based on diffusion tensor imaging in an ICBM template. NeuroImage 40, $570-582$.

Mori, S., Zhang, J., 2006. Principles of diffusion tensor imaging and its applications to basic neuroscience research. Neuron 51 (5), 527539

Murgasova, M., Dyet, L., Edwards, D., Rutherford, M., Hajnal, J., Rueckert, D., 2007. Segmentation of brain MRI in young children. Acad Radiol 14

Pajevic, S., Pierpaoli, C., et al., 1999. Color schemes to represent the orientation of anisotropic tissues from diffusion tensor data: application to white matter fiber tract mapping in the human brain. Magnetic Resonance in Medicine 42 (3), 526-540.

Pfefferbaum, A., Mathalon, D., Sullivan, E., Rawles, J., Zipursky, R., Lim, K., 1994. A quantitative magnetic resonance imaging study of changes in brain morphology from infancy to late adulthood. Archives of Neurology 51 (9), 874-887.

Pierpaoli, C., Basser, P. J., 1996. Toward a quantitative assessment of diffusion anisotropy. Magnetic resonance in Medicine 36 (6), 893-906.

Rutherford, M. (Ed.), 2002. MRI of the Neonatal Brain. WB Saunders.

Sadeghi, N., Prastawa, M., Fletcher, P., Gilmore, J., Lin, W., Gerig, G., 2012. Statistical growth modeling of longitudinal dt-mri for regional characterization of early brain development. In: Proceedings of the 2012 IEEE International Symposium on Biomedical Imaging: From Nano to Macro. pp. 1507-1510.

Sadeghi, N., Prastawa, M., Fletcher, P. T., Wolff, J., Gilmore, J. H., Gerig, G., 2013. Regional characterization of longitudinal dt-mri to study white matter maturation of the early developing brain. Neuroimage.

Sadeghi, N., Prastawa, M., Gilmore, J., Lin, W., Gerig, G., 2010 Spatio-temporal analysis of early brain development. In: Proceedings IEEE Asilomar Conference on Signals, Systems and Computers. pp. $777-781$.

van Gelderen, P., de Vleeschouwer, M. H., DesPres, D., Pekar, J., van Zijl, P., Moonen, C. T., 1994. Water diffusion and acute stroke. Magnetic resonance in medicine 31 (2), 154-163.

Xue, H., Srinivasan, L., Jiang, S., Rutherford, M., Edwards, A., Rueckert, D., Hajnal, J. V., 2007. Automatic cortical segmentation in the developing brain. In: IPMI. pp. 257-269.

Yakovlev, P., Lecours, A., 1967. The myelogenetic cycles of regional maturation of the brain. In: Minkowski A, editor. Regional development of the brain in early life. Blackwell Scientific, Oxford, UK, pp. 3-70. 